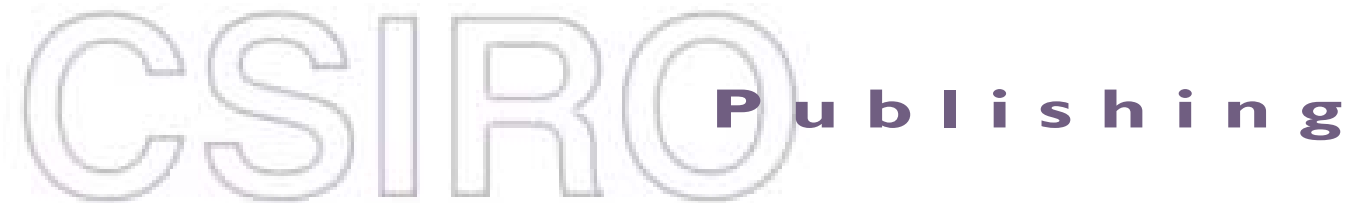

\section{Publications of the Astronomical Society of Australia}

Volume 19, 2002

(C) Astronomical Society of Australia 2002

An international journal of astronomy and astrophysics

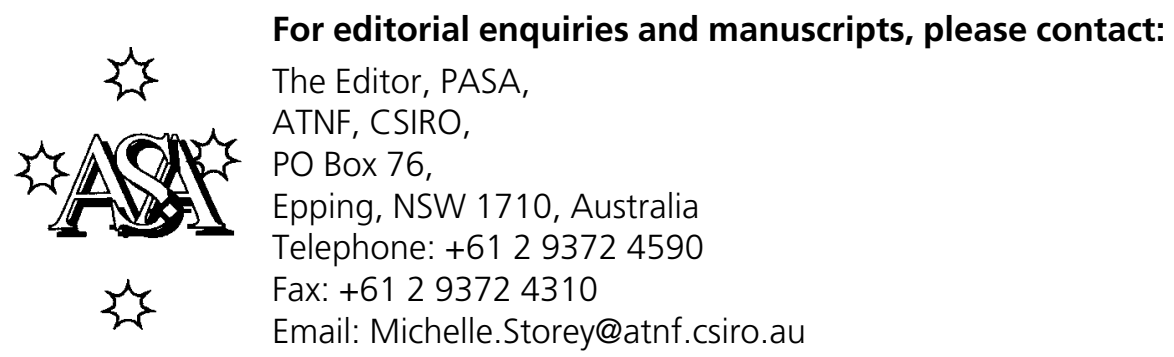

For general enquiries and subscriptions, please contact: CSIRO Publishing PO Box 1139 (150 Oxford St)

Collingwood, Vic. 3066, Australia

Telephone: +6139662 7666

Fax: +61 396627555

Email: publishing.pasa@csiro.au

C S I RO

PUBLISHING Published by CSIRO Publishing

for the Astronomical Society of Australia

www.publish.csiro.au/journals/pasa 


\title{
The Parsec-scale Jets of Extragalactic TeV Gamma-ray Sources
}

\author{
B. G. Piner ${ }^{1}$, P. G. Edwards ${ }^{2}$, S. Fodor ${ }^{1}$ and T. A. Rector ${ }^{3}$ \\ ${ }^{1}$ Department of Physics and Astronomy, Whittier College, 13406 E. Philadelphia St., \\ Whittier, CA 90608, USA \\ gpiner@whittier.edu \\ ${ }^{2}$ ISAS, Yoshinodai 3-1-1, Sagamihara, Kanagawa 229-8510, Japan \\ ${ }^{3}$ NOAO, 950 North Cherry Avenue, Tucson, AZ 85719, USA
}

Received 2001 July 31, accepted 2002 March 28

\begin{abstract}
We present results of VLBI monitoring observations of the blazars detected by ground-based $\mathrm{TeV}$ gamma-ray telescopes. Preliminary results have shown that the apparent speeds of the parsec-scale jet features in these sources are predominantly subluminal, and significantly slower than those in the GeVpeaked (EGRET) gamma-ray blazars. Here we add results from additional sources and additional epochs that increase the statistical significance of this comparison. These slower speeds may indicate rapid deceleration of the jets in $\mathrm{TeV}$ blazars between the TeV gamma-ray emitting scales and VLBI scales.
\end{abstract}

Keywords: BLLacertae objects: individual (Mrk 501, 1ES 1959+650, PKS 2155-304, 1ES 2344+514) galaxies: active — galaxies: jets — radio continuum: galaxies — radiation mechanisms: non-thermal

\section{Introduction}

Detectable fluxes of $\mathrm{TeV}\left(\sim 1 \times 10^{12} \mathrm{eV}\right) \gamma$-rays have been reported for eight AGN: Mrk 421 (Punch et al. 1992), Mrk 501 (Quinn et al. 1996), 1ES 2344+514 (Catanese et al. 1998), PKS 2155-304 (Chadwick et al. 1999), 1ES 1959+650 (Nishiyama et al. 1999), 3C 66A (Neshpor et al. 1998), BL Lac (Neshpor et al. 2001), and 1ES 1426+428 (Horan et al. 2001). At present only Mrk 421 and Mrk 501 have been confirmed by independent groups as $\mathrm{TeV}$ gamma-ray sources. Short-timescale variability of the $\gamma$-ray emission implies high Doppler factors in the $\gamma$-ray emitting regions for at least some of these sources (see the review of Catanese \& Weekes 1999). We have analysed multi-epoch VLBI observations of five of these $\mathrm{TeV}$ blazars, to determine if high Doppler factors are also evident in the parsec-scale jet structure. We have also recently completed a first-epoch observation of 1ES $1426+428$, but have not observed 3C66A, whose high redshift makes it an unlikely $\mathrm{TeV}$ source.

\section{Observations}

Mrk 421 and Mrk 501 both have a significant number of archival VLBI observations. For these sources, we have combined this archival VLBI data (from the U.S. Naval Observatory Radio Reference Frame Image Database [e.g. Fey \& Charlot 1997] and from the VLBA $2 \mathrm{~cm}$ survey [e.g. Kellermann et al. 1998]) with our own VLBA and VSOP data. All archival data was independently imaged and/or model fitted by us, starting from the calibrated $(u, v)$ data. The other three sources that we studied (1ES 1959+650, PKS 2155-304, and 1ES 2344+514) have fainter VLBI flux densities $(\sim 100 \mathrm{mJy})$ and had not been previously studied with VLBI. We monitored these sources with the NRAO VLBA at $15 \mathrm{GHz}$ during 1999 and 2000. Some of these observations are also discussed by, e.g. Edwards et al. (2001), who present more information on the kiloparsec-scale structure of these sources. These observations will be described in detail, including complete sets of images and model fits, in forthcoming publications (Edwards \& Piner, in preparation, for Mrk 501; Piner et al., in preparation, for 1ES 1959+650, PKS 2155-304, and 1ES 2344+514).

\section{Results on Individual Sources}

\subsection{Mrk 421}

Analysis of 30 VLBI images of Mrk 421 at 15 epochs is presented by Piner et al. (1999). The apparent speeds of three components were measured, and all were found to be subluminal (see Table 1). These measured speeds differed from apparent superluminal speeds obtained from 3 epochs of VLBI data from the early 1980s by Zhang \& Bååth (1990). With the benefit of hindsight, it appears the

Table 1. Apparent component speeds

\begin{tabular}{lcr}
\hline Source & Component $^{a}$ & Speed $^{b}(c)$ \\
\hline Mrk 421 & C4 & $0.2 \pm 0.3$ \\
& C5 & $0.3 \pm 0.1$ \\
Mrk 501 & C6 & $-0.1 \pm 0.1$ \\
& C1 & $0.1 \pm 0.2$ \\
& C2 & $0.8 \pm 0.1$ \\
1ES 1959+650 & C3 & $0.3 \pm 0.1$ \\
& C4 & $0.0 \pm 0.1$ \\
PKS 2155-304 & C2 & $0.1 \pm 1.1$ \\
1ES 2344+514 & C1 & $-0.2 \pm 0.8$ \\
& C1 & $4.6 \pm 3.8$ \\
& C2 & $0.3 \pm 0.8$ \\
& C3 & $-0.2 \pm 0.8$ \\
\hline
\end{tabular}

${ }^{a}$ Components are numbered $\mathrm{C} 1, \mathrm{C} 2, \ldots$ from the outermost component inward.

${ }^{b}$ For $H_{0}=65 \mathrm{~km} \mathrm{~s}^{-1} \mathrm{Mpc}^{-1}$. 

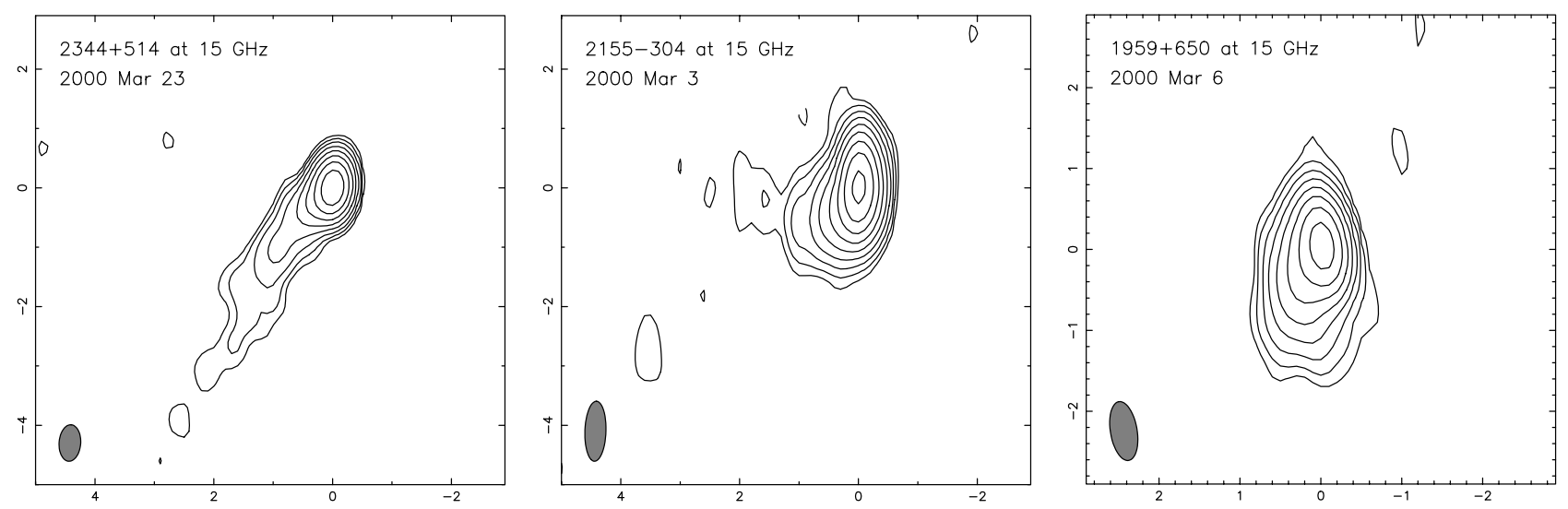

Figure $115 \mathrm{GHz}$ VLBA images of 1ES 2344+514, PKS 2155-304, and 1ES 1959+650. The uniformly weighted restoring beams have FWHM sizes of $0.6 \times 0.4$ mas, $1.0 \times 0.4$ mas, and $0.7 \times 0.3$ mas, respectively. The lowest contour in each image has been set to 3 times the rms noise level in the image, and is equal to $0.35,0.62$, and $0.45 \mathrm{mJy}_{\text {beam }}^{-1}$, respectively. Successive contours are each a factor of 2 higher. The peak flux densities are 85,194 , and $85 \mathrm{mJy} \mathrm{beam}^{-1}$ for the three images, respectively. The axes are labelled in milliarcseconds.

uncertainties in component positions assigned by Zhang \& Bååth (1990) were too small. With larger assumed errors, these earlier observations are consistent with those of Piner et al. (1999).

\subsection{Mrk 501}

For Mrk 501, we have analysed a total of 11 epochs of VLBI data: four from the USNO RRFID, six from the VLBA $2 \mathrm{~cm}$ survey, and one VSOP observation. A total of four jet components can be identified, with apparent speeds ranging up to $0.8 c$ (see Table 1).

\subsection{IES $1959+650$}

We observed 1ES $1959+650$ with the VLBA at 3 epochs at $15 \mathrm{GHz}$ between $2000 \mathrm{March}$ and 2000 July. All three epochs have been imaged, an image from the first epoch is shown in Figure 1. Two jet components whose positions are consistent with no apparent motion were identified at all three epochs (see Table 1).

\subsection{PKS 2155-304}

PKS 2155-304 is a well-known X-ray selected BL Lac object. We observed this source with the VLBA at 3 epochs at $15 \mathrm{GHz}$ between 2000 March and 2000 June. All three epochs have been imaged, and an image from the first epoch is shown. We identified one component with an apparent speed of $4.6 \pm 3.8 c$. Additional epochs would help to reduce the large error on this measurement.

\section{$3.51 E S 2344+514$}

We observed 1ES $2344+514$ four times with the VLBA at $15 \mathrm{GHz}$ between 1999 October and 2000 March. All four epochs have been imaged, an image from the latest epoch is shown in Figure 1. A total of three components were identified by model fitting, at each of the four epochs, with apparent speeds ranging up to $1.3 c$ (see Table 1).

\section{Discussion}

The TeV ('blue') blazars appear to have substantially slower apparent speeds than the GeV ('red') blazars. All but one of the radio components we have measured are subluminal or only slightly superluminal. We have compared the component speeds we have measured in $\mathrm{TeV}$ blazars to the apparent speeds measured in the jets of $\mathrm{GeV}$ (EGRET) blazars (Jorstad et al. 2001). A KolmogorovSmirnov test shows the two distributions are different with a significance of $2 \times 10^{-5}$. These slow apparent speeds must somehow be reconciled with the high Doppler factors demanded by rapid TeV variability.

It is possible for low apparent speeds to be caused by a small viewing angle of the parsec-scale jet. However, this requires very small viewing angles (often $\ll 1$ degree), and in this case other radio properties indicative of a large Doppler factor such as extreme radio variability and very high apparent brightness temperatures should also be observed, and they are not. We consider the most likely explanation to be that the jet is physically slowing down between the region where $\mathrm{TeV} \gamma$-rays are generated and the scales mapped by VLBI.

A mechanism to decelerate TeV blazar jets is described by Marscher (1999). If the jet is electron-positron with a sufficiently flat electron index, then most of the energy will be carried in the highest energy electrons that lose energy very efficiently to synchrotron and inverse Compton emission, producing the $\mathrm{X}$-rays and $\mathrm{TeV} \gamma$-rays, and dumping most of the energy and momentum close to the base of the jet. If deceleration of $\mathrm{TeV}$ blazar jets is confirmed by additional work, it may be an important argument for light (electron-positron) jets in these sources.

\section{Acknowledgments}

We thank Ken Kellermann and Alan Fey for providing us with VLBI data on Mrk 421 and Mrk 501. This research has made use of the United States Naval Observatory (USNO) Radio Reference Frame Image Database 
(RRFID). The National Radio Astronomy Observatory is a facility of the National Science Foundation operated under cooperative agreement by Associated Universities, Inc.

\section{References}

Catanese, M., \& Weekes, T. C. 1999, PASP, 111, 1193

Catanese, M., et al. 1998, ApJ, 501, 616

Chadwick, P. M., et al. 1999, ApJ, 513, 161

Edwards, P. G., Piner, B. G., Fodor, S., \& Rector, T. A. 2001, in High Energy Gamma-Ray Astronomy, AIP Conf. Series 558, eds F. A. Aharonian, \& H. J. Völk (San Francisco: ASP), 672

Fey, A. L., \& Charlot, P. 1997, ApJS, 111, 95

Horan, D., et al. 2001, in Proc. XXVII Int. Cosmic Ray Conf. vol. 7, eds M. Simon, E. Lorenz, \& M. Pohl (Hamburg, Germany: IUPAP), 2622
Jorstad, S. G., Marscher, A. P., Mattox, J. R., Wehrle, A. E., Bloom, S. D., \& Yurchenko, A. V. 2001, ApJS, 134, 181

Kellermann, K. I., Vermeulen, R. C., Zensus, J. A., \& Cohen, M. H. 1998, AJ, 115, 1295

Marscher, A. P. 1999, APh, 11, 19

Neshpor, Yu. I., et al. 1998, AstL, 24, 134

Neshpor, Yu. I., et al. 2001, ARep, 45, 249

Nishiyama, T., et al. 1999, Proc. XXVI Int. Cosmic Ray Conf., Salt Lake City, eds D. Kieda, M. Salamon, \& B. Dingus, 3, 370

Piner, B. G., Unwin, S. C., Wehrle, A. E., Edwards, P. G., Fey, A. L., \& Kingham, K. A. 1999, ApJ, 525, 176

Punch, M., et al. 1992, Nature, 358, 477

Quinn, J., et al. 1996, ApJ, 456, L83

Zhang, F. J., \& Bååth, L. B. 1990, A\&A, 236, 47 